\title{
SIMCE: PASADO, PRESENTE Y FUTURO DEL SISTEMA NACIONAL DE EVALUACIÓN
}

\author{
Juan Bravo
}

Resumen: En este artículo se describen las principales transformaciones que ha experimentado el Sistema Nacional de Medición de la Calidad de la Educación (SIMCE) desde su creación en 1988 hasta nuestros días, y se analizan los trascendentales desafíos que deberá enfrentar el sistema de evaluación en un futuro próximo, producto de la entrada en vigencia de la Ley de Aseguramiento de la Calidad de la Educación (2011) que reconfigura la institucionalidad del sistema escolar.

Palabras clave: educación, evaluaciones externas, estándares, rendición de cuentas.

Recibido: abril 2011; aceptado: mayo 2011.

\section{SIMCE: PAST, PRESENT AND FUTURE OF THE NATIONAL EVALUATION SYSTEM Juan Bravo}

Abstract: This article describes the main transformations that SIMCE (the Chilean national evaluation system of learning achievements) has undergone since its creation

Juan Bravo M. Magíster en Ciencias de la Educación, Pontificia Universidad Católica de Chile. Director Sistema Nacional de Medición de la Calidad de la Educación (SIMCE), Ministerio de Educación (juan.bravo@mineduc.cl). 
in 1988. The transcendental challenges that the evaluation system must face in the near future are analyzed in light of the entry into force of regulations that redesign the framework of the school system.

Keywords: education, external evaluation, standards, accountability.

Received: April 2011; accepted: May 2011.

\section{Introducción}

$\mathrm{L}$

a evaluación de logros de aprendizaje de los estudiantes es un factor clave para determinar los avances en materia de calidad y equidad educacional, así como para identificar y superar las debilidades e insuficiencias de los sistemas educativos nacionales.

En el contexto latinoamericano, Chile ha sido pionero en la creación y consolidación de un sistema de evaluación de logros de aprendizaje, dado que a través de una serie de mediciones a lo largo de los años ha sido posible determinar los logros de aprendizaje de los estudiantes y relacionarlos con el contexto escolar y social en el que se producen, de manera de aportar evidencia empírica para orientar la toma de decisiones de política educativa. Adicionalmente, los resultados de las evaluaciones han permitido retroalimentar las prácticas pedagógicas y de gestión en los establecimientos, mediante la distribución de reportes para docentes y directivos, la organización de seminarios de difusión de los resultados y la realización de jornadas de análisis en los establecimientos educacionales.

En la actualidad, la labor de evaluar los logros de aprendizaje de los estudiantes recae directamente en el Ministerio de Educación (Mineduc), institución que cumple esta tarea a través del componente evaluación de la Unidad de Curriculum y Evaluación del SIMCE ${ }^{1}$.

Este artículo tiene como objetivo describir las principales transformaciones que ha experimentado el sistema nacional de evaluación

${ }^{1}$ La sigla empleada para identificar al sistema nacional de evaluación de logros de aprendizaje es SIMCE (Sistema de Medición de la Calidad de la Educación); no obstante, su propósito principal es la medición de resultados de aprendizaje. 
SIMCE desde su creación, y analizar los desafíos que enfrentará en un futuro próximo, producto de la entrada en vigencia de la normativa que reconfigura la institucionalidad del sistema escolar. Para esto, en la primera parte del documento se describe la historia del SIMCE, desde su creación hasta nuestros días; a continuación se detalla la situación actual del sistema, y por último, en la tercera parte, se analizan los principales desafíos que deberá enfrentar en un futuro inmediato.

\section{Un sistema que se enmarca dentro de una larga tradición evaluativa}

\subsection{Los orígenes del sistema nacional de evaluación, SIMCE}

Los orígenes del SIMCE se remontan a la década de los 60, cuando en 1968 se rinde la primera Prueba Nacional, destinada a medir los logros de aprendizaje de los estudiantes en $8^{\circ}$ año básico. Dicha prueba se aplicó anualmente hasta 1971 y posteriormente se descontinuó. En 1982 se implementó el Programa de Evaluación del Rendimiento Escolar (PER) para $4^{\circ}$ y $8^{\circ}$ año básico; este programa fue diseñado y ejecutado por el Departamento de Investigación y Tecnología de la Universidad Católica (DICTUC), mediante un convenio con el Mineduc. El PER se entendió como una herramienta para hacer efectiva la descentralización de la educación, al trasladar la iniciativa sobre el mejoramiento educativo a los docentes, directivos, y padres y apoderados, proporcionando información sobre los resultados de aprendizaje de los estudiantes (Himmel, 1997). Entre los años 1985 y 1986 se crea el Sistema de Evaluación de la Calidad de la Educación (SECE), a cargo del Centro de Perfeccionamiento, Experimentación e Investigaciones Pedagógicas (CPEIP) del Ministerio de Educación, con el objetivo de analizar los datos generados por el PER (Román, 1999).

En 1988 se puso en marcha el SIMCE, sistema destinado en principio a evaluar el rendimiento escolar y la calidad de la educación impartida en los niveles $4^{\circ}$ y $8^{\circ}$ básico de todos los establecimientos educacionales del país. Entre sus objetivos originales destacaban:

- Proporcionar asistencia al Ministerio de Educación en su tarea normativa y en la supervisión del sistema. 
- Brindar apoyo a las autoridades regionales y locales en la supervisión, además de prestarles apoyo técnico.

- Estimar la calidad de la educación impartida en cada establecimiento educacional; comparar dichos resultados, buscar los factores explicativos y evaluar los resultados de los programas pedagógicos.

- Orientar la actividad de perfeccionamiento docente, las acciones de supervisión y la asignación de recursos.

Finalmente, en 1992 el Mineduc asume el control del SIMCE, basando esta iniciativa en la Ley Orgánica Constitucional de Enseñanza (LOCE) de marzo de 1990, la cual establece que: “Corresponderá al Ministerio de Educación Pública diseñar los instrumentos que permitan el establecimiento de un sistema para la evaluación periódica, tanto de la enseñanza básica como media, de los objetivos fundamentales y contenidos mínimos de esos niveles" (Decreto Ley N 40, Mineduc, 1990). Esta normativa también determina las principales características del sistema, a saber: las pruebas deben evaluar el marco curricular vigente, se debe evaluar al finalizar cada ciclo escolar, y se deben hacer públicos los resultados de cada establecimiento.

\subsection{La institucionalización del sistema y el surgimiento de una cultura evaluativa $^{2}$}

Tal como se señaló previamente, a partir de 1992 el SIMCE se institucionaliza en el Mineduc; no obstante, su instalación en el sistema y el reconocimiento por parte de los establecimientos no concluye sino hasta mediados de la década de los 90, cuando comienzan a publicarse en un diario de circulación nacional los resultados de cada establecimiento, y tanto los docentes como los directivos reconocen que el SIMCE es un medio útil para la gestión pedagógica (Himmel, 1997). De hecho, desde el comienzo de la publicación de los resultados, esta se convirtió en un hito no sólo para los establecimientos educacionales,

${ }^{2}$ La "cultura evaluativa" implica la posesión de competencias profesionales en evaluación educativa, el liderazgo organizativo y el uso de la información producto de la evaluación en la labor cotidiana. En el caso del SIMCE, esta "cultura evaluativa" se ha ido desarrollando gradualmente en el tiempo, lo que ha contribuido a consolidar este sistema. 
sino también para la opinión pública, debido a la gran cobertura que le otorgan los medios de comunicación masiva.

Entre 1992 y 1997 las evaluaciones censales se concentraron en logros académicos de Castellano y Matemáticas (la evaluación de Ciencias Naturales e Historia y Geografía se realizaba muestralmente), recogiéndose también información sobre desarrollo personal de los alumnos, actitudes hacia el medio ambiente, desarrollo de la creatividad, y sobre la aceptación de la labor educacional por parte de los padres, como también información técnico-administrativa de los establecimientos (Meckes, 2003).

El modelo de medición utilizado durante este periodo fue la Teoría Clásica de las pruebas; esta metodología permitió determinar y reportar el porcentaje promedio de respuestas correctas de los estudiantes sobre el total de preguntas presentadas en cada prueba, a nivel de establecimiento. Los resultados obtenidos eran comparables entre establecimientos o agrupaciones de establecimientos dentro de un mismo año; pero no eran comparables entre distintos años, debido a que la construcción de las pruebas y su metodología de análisis no consideraban los resguardos técnicos requeridos para ello. Sin embargo, desgraciadamente los puntajes comenzaron a ser utilizados para establecer comparaciones entre años, a pesar de estas limitaciones.

\subsection{La consolidación del sistema y la Comisión SIMCE}

En 1998, el SIMCE cambió su dependencia dentro del Ministerio de Educación y de ser una unidad que dependía directamente de la Subsecretaría de Educación, pasó a formar parte de la Unidad de Currículum y Evaluación (UCE). En un primer período como parte de la UCE — comprendido entre los años 1998 y 2003 - se priorizaron tres líneas de desarrollo del SIMCE (Meckes, 2003), a saber:

- Alinear las pruebas nacionales con la reforma curricular y comunicar los nuevos lineamientos de la evaluación a los docentes, con el propósito de contribuir a la implementación del nuevo currículum en la sala de clases.

- Modernizar las metodologías de análisis de la evaluación, posibilitando las comparaciones de los resultados entre años, y el análisis de tendencias a lo largo del tiempo. 
- Dar impulso a la participación de Chile en diversos estudios internacionales con el propósito de situar el rendimiento de los estudiantes chilenos en un contexto internacional y retroalimentar el diseño curricular.

En primer lugar, las pruebas se orientaron a la evaluación del nuevo marco curricular, enfatizando la medición de habilidades cognitivas de orden superior, mediante la incorporación de preguntas orientadas a la medición de éstas, tales como las preguntas abiertas o de desarrollo que se incorporaron en todas las pruebas. Además, se puso énfasis en la publicación de ejemplos de preguntas, puesto que la difusión de ejemplos de preguntas y respuestas de los estudiantes, en el caso de las preguntas abiertas, constituía una forma de comunicar a los docentes cuáles eran las expectativas de aprendizaje asociadas al marco curricular.

Otro de los avances importantes del SIMCE consistió en la actualización del modelo de medición utilizado, ya que en 1998 se cambió el modelo de medición, reemplazando el uso de la Teoría Clásica por la Teoría de Respuesta al Ítem (TRI), para puntuar las pruebas. La adopción de esta nueva metodología facilitó la comparación de resultados entre años, así como la descripción de niveles de desempeño de los estudiantes, a partir de los resultados.

También se dio impulso a la participación de Chile en diversos estudios internacionales, organizados por la Asociación Internacional de Educación (IEA), la Organización para la Cooperación y el Desarrollo Económico (OCDE), y la Oficina Regional de Unesco. Así el SIMCE llevó adelante el Primer Estudio Regional Comparativo y Explicativo (PERCE) del Laboratorio Latinoamericano de la Calidad de la Educación dependiente de Unesco, en 1997; las primeras aplicaciones del Estudio Internacional de Tendencias en Matemáticas y Ciencias (TIMSS 1999 y 2003) y del Estudio Internacional de Educación Cívica (ICCS 1999), dependientes de la IEA; y la primera aplicación del Programa Internacional de Evaluación de Estudiantes (PISA) dependiente de la OCDE, en 2000.

Adicionalmente es importante mencionar que a partir de la evaluación del año 2000 se comenzaron a publicar los resultados de los establecimientos en la prensa y en la página web, comparándolos con los puntajes promedio obtenidos por aquellos establecimientos con similares características socioeconómicas. Esto con el propósito de contar 
con un referente de comparación que permitiera distinguir el aporte de variables propias del desempeño del establecimiento y aquellas que son menos controlables por estos.

\subsubsection{La Comisión SIMCE}

En 2003, el Ministerio de Educación convocó un comité de expertos de diversos ámbitos institucionales relevantes para el sistema educacional chileno, para constituir la "Comisión para el Desarrollo y Uso del Sistema de Medición de la Calidad de la Educación" (Comisión SIMCE), con el objetivo de elaborar un diagnóstico sobre el sistema nacional de evaluación y la información que este otorga, y proporcionar alternativas de mejoramiento orientadas a optimizar su impacto sobre la calidad de los aprendizajes (Meckes, 2003). La Comisión fue una primera instancia de reflexión sobre los alcances que tenía el SIMCE y sobre la necesidad de responder a las demandas del sistema educacional chileno en el ámbito de la evaluación de aprendizajes.

El diagnóstico que entregó la Comisión permitió corroborar que el SIMCE era un sistema metodológicamente sólido, que gozaba de gran credibilidad entre los distintos actores educativos, pero que era utilizado principalmente para focalizar recursos, instalar incentivos vinculados a los resultados y evaluar el impacto de las políticas (Mineduc, 2003). Los usos pedagógicos de la información de resultados resultaban muy restringidos, dado que la información reportada a los docentes muchas veces carecía de significado para orientar sus prácticas. Además, se detectó que el uso de la información de resultados, por parte de los padres y apoderados, era prácticamente nulo. Finalmente, la Comisión recogió la preocupación por el impacto negativo de las prácticas de selección $\mathrm{y}$ de exclusión de estudiantes con bajo rendimiento, como estrategias para mejorar los resultados del SIMCE y, asimismo, se detectó cierta tendencia de los establecimientos a concentrar sus mejores recursos y esfuerzos en las generaciones evaluadas por el SIMCE, en desmedro de las que no lo eran.

En cuanto a los propósitos del SIMCE, la Comisión recomendó que el sistema debiera obtener y proveer información sobre los aprendizajes logrados por los estudiantes para:

- $\quad$ permitir el monitoreo de resultados a nivel nacional y orientar decisiones de política educativa; 
- retroalimentar las prácticas pedagógicas y de gestión de los docentes y directivos, con el fin de mejorar los procesos de enseñanza y aprendizaje;

- comunicarlos a las familias de los estudiantes y a la comunidad con el objetivo de promover el compromiso responsable de los involucrados y permitir la rendición de cuentas de sostenedores, directivos y docentes.

La Comisión también entregó una serie de recomendaciones orientadas a favorecer un mejor uso de los resultados del SIMCE por parte de los actores educativos; a aumentar el impacto de la evaluación en el sistema escolar, mediante un aumento de su periodicidad; y a entregar información que facilite la implementación de incentivos más justos y válidos. En primer lugar, sugirió reportar los resultados en relación con expectativas nacionales de logro o estándares de desempeño, para otorgar un mayor significado y utilidad pedagógica a los resultados del SIMCE. También recomendó establecer canales de información más directos, para informar eficazmente a los padres y apoderados de los estudiantes acerca de los resultados obtenidos, en cada establecimiento. Por otra parte, la Comisión sugirió evaluar más niveles educativos cada año e incorporar nuevas áreas curriculares a la medición, como por ejemplo Inglés y Educación Tecnológica, con el propósito de promover una implementación más amplia del currículum, por parte de los establecimientos. Por último, recomendó asociar consecuencias a los avances alcanzados por los establecimientos, a partir de los resultados obtenidos por un grupo de estudiantes entre un nivel evaluado y el siguiente, utilizando indicadores de progreso educativo o valor agregado.

También cabe destacar que en 2004 un grupo de especialistas de la OCDE realizó una evaluación de las políticas educacionales de Chile (OCDE, 2004b), que derivó en una serie de recomendaciones de mejora para el SIMCE. En general, estas recomendaciones fueron coincidentes con las recomendaciones que había realizado la Comisión SIMCE, por lo que, en conjunto con estas últimas, orientaron el desarrollo del sistema durante los años siguientes.

Por último, es importante aclarar que las recomendaciones de mejoramiento, tanto de la Comisión como de la revisión de la OECD, 
estuvieron fundadas en una sugerencia de base referida a conservar las principales características del SIMCE, es decir, mantener:

- $\quad$ su foco central en los aprendizajes,

- la calidad de censal de la medición de $4^{\circ}, 8^{\circ}$ y $2^{\circ}$ medio,

- la publicación de los resultados de cada establecimiento,

- la confidencialidad de los puntajes individuales,

- el reporte de información para contextualizar los resultados y la entrega de información sobre equidad en los logros de aprendizaje,

- la participación en mediciones internacionales,

- el acceso a la información para fines de investigación.

\subsection{La evolución del sistema post-Comisión SIMCE}

Entre 2005 y 2010 se implementó la mayor parte de las recomendaciones surgidas de la Comisión SIMCE y de la evaluación de políticas educacionales realizada por la OCDE. No obstante, cabe señalar que antes de la ejecución de estas recomendaciones se reestructuró internamente la organización, para operar de manera más eficiente en relación con las nuevas demandas emanadas de dichas instancias. En este contexto, el SIMCE incorporó a su estructura a la hasta ese entonces independiente Área de Estudios Internacionales, y creó dos instancias directamente dependientes de la Coordinación Nacional: la Coordinación Técnica (a cargo de los equipos de evaluaciones nacionales e internacionales, esto es, los equipos disciplinarios, de medición, y de análisis y comunicación de resultados) y la Coordinación de Gestión (a cargo de programar y monitorear la ejecución de los procesos y su correspondiente control de calidad, además de ser la contraparte de la UPLAG, en los procesos operativos del SIMCE).

En el período 2005-2010 destacó nítidamente la realización de una serie de acciones que permitieron reportar resultados según estándares de desempeño o niveles de logro, como se les denominó en el ámbito nacional. En efecto, durante este período se implementaron progresivamente pruebas referidas a niveles de logro en $4^{\circ}$ año básico y $8^{\circ}$ año básico; lo que representó un gran avance para el sistema, puesto que implicó un cambio de foco en la entrega de información sobre resultados SIMCE para los docentes y directivos, poniendo mayor énfasis en 
los aprendizajes que mostraban los estudiantes que en los promedios de los puntajes obtenidos (Meckes, 2007). Además, entre 2007 y 2010 se publicaron los documentos de descripción de los niveles de logro (NL) de Lectura, Educación Matemática, Comprensión del Medio Natural y Comprensión del Medio Social y Cultural, que incluyen orientaciones para su uso, tanto en la interpretación de los resultados como en el diagnóstico de los aprendizajes de los estudiantes evaluados.

Otra acción destacable del periodo se refiere a la intensificación de la frecuencia de la medición nacional y a permitir el seguimiento de una misma cohorte de estudiantes a lo largo de su trayectoria escolar. En este ámbito, en 2005 se inició la medición anual de $4^{\circ}$ año básico, que en los años sucesivos y hasta la fecha es acompañada por las pruebas de $2^{\circ}$ año medio y de $8^{\circ}$ año básico, alternadamente. En 2009, se contó con la primera cohorte que había rendido la prueba en $4^{\circ}$ año básico y $8^{\circ}$ año básico, posibilitando la realización de un primer estudio de indicadores de progreso educativo y la entrega de recomendaciones para su eventual incorporación al reporte de resultados de $8^{\circ}$ año básico. Ese mismo año se realizó un escalamiento vertical entre las pruebas de $4^{\circ}$ y $8^{\circ}$ básico de Lectura y Educación Matemática, referidas a los $\mathrm{NL}$, lo que permitió determinar el avance alcanzado por los estudiantes (Montt et al., 2010).

Respecto del aumento de la accesibilidad de las pruebas SIMCE, también se realizaron acciones importantes: en 2008 se realizó un estudio piloto para evaluar a estudiantes de $4^{\circ}$ año básico con discapacidad visual y auditiva (estudiantes ciegos, con baja visión y sordos). El éxito de este estudio permitió que en 2009 y 2010 se aplicara esta evaluación especial, en Lectura y Matemática, de manera censal en la Región Metropolitana y en Valparaíso.

En otro ámbito y con el objeto de promover el uso de los resultados de medición por parte de los distintos actores educativos, en 2006 se realizó un estudio sobre acceso, comprensión y uso de los resultados del SIMCE, y conforme a ello, posteriormente se introdujeron modificaciones al informe para docentes y directivos, y al informe para padres y apoderados que se había comenzado a distribuir ese mismo año. Además, se elaboró un nuevo informe destinado a académicos y autoridades de gobierno, al que en forma paulatina se incorporó la información relativa a análisis descriptivos y explicativos de factores asociados a 
los logros de aprendizaje de los estudiantes. Asimismo, se estableció un vínculo cooperativo permanente con la División de Educación General (DEG), para fortalecer la jornada de análisis de resultados que coordinaba y supervisaba dicho organismo (Montt et al., 2010).

Otro hito importante dentro del período 2005-2010 fue la incorporación de nuevas áreas curriculares al SIMCE, dado que se realizaron por primera vez: una evaluación de la producción escrita, en $4^{\circ}$ año básico; una evaluación de Educación Física, en $8^{\circ}$ año básico; y una evaluación de Inglés, en $3^{\text {er }}$ año medio.

La promoción de un mayor uso de los resultados de estudios internacionales fue otra acción destacada del período. En este contexto, en 2006 se aplicó el segundo Estudio Regional Comparativo y Explicativo (SERCE); en 2009 el segundo el Estudio Internacional de Educación Cívica; y en 2006, la segunda aplicación del Programa Internacional de Evaluación de Estudiantes (PISA). Posteriormente se convocó a diversos investigadores nacionales para que realizaran estudios sobre la base de dichos resultados, orientados hacia la propuesta de políticas educativas en diversos ámbitos. Estos estudios fueron compilados en una publicación SIMCE a fines de 2009. Ese mismo año también se aplicó la prueba PISA 2009, con acento en Lectura, que incorporó, por primera vez, una evaluación electrónica sobre comprensión de textos.

Finalmente, cabe destacar que durante el período se realizaron diversas acciones de fortalecimiento institucional. En 2007 y 2009, con el objeto de formar y mantener los recursos humanos, se dictaron cursos de capacitación a los miembros de todos los equipos de trabajo. Durante 2008 se adoptó una política de remuneraciones que permitió nivelar las remuneraciones, tanto dentro de los equipos como entre los coordinadores de las distintas áreas. En 2009 se elaboró un organigrama y mapa de funciones del SIMCE (Montt et al., 2010).

\section{La situación actual del sistema nacional de evaluación}

Después de más de dos décadas de funcionamiento ininterrumpido el SIMCE es un componente clave del sistema educativo nacional que ha contribuido al mejoramiento de la calidad y equidad de la educación, informando sobre el desempeño de los estudiantes en diferentes 
sectores de aprendizaje del currículum nacional y relacionándolo con el contexto escolar y social en el que estos aprenden.

Para lograr este objetivo, el SIMCE ha desarrollado instrumentos de evaluación que consisten en pruebas y cuestionarios de contexto. Las pruebas evalúan el logro de los Objetivos Fundamentales y Contenidos Mínimos Obligatorios (OF-CMO) del marco curricular vigente en diferentes sectores de aprendizaje. En su mayoría, se aplican anualmente a nivel nacional y son de carácter censal. Los cuestionarios de contexto, por su parte, son respondidos por cada uno de los estudiantes evaluados, por sus padres o apoderados y por sus profesores. Estos instrumentos permiten recoger información acerca de todos ellos, la que se utiliza para realizar análisis de factores asociados y contextualizar los resultados obtenidos por los estudiantes en las pruebas SIMCE.

Además de las mediciones nacionales, el SIMCE ha gestionado e implementado en Chile la aplicación de evaluaciones internacionales cuyos resultados permiten situar a nuestro país en el contexto regional o global, y de este modo determinar los niveles de desempeño de los estudiantes chilenos respecto de estándares internacionales.

\subsection{Visión y misión institucional}

En la actualidad, la visión del sistema apunta a que éste se transforme en "una institución líder a nivel nacional e internacional en evaluación educacional, que informa eficaz y oportunamente a los distintos actores educativos, acerca de los resultados de aprendizaje del sistema escolar y sus factores asociados, contribuyendo a asegurar una educación de calidad para todos los estudiantes del país".

En cuanto a su misión institucional, el SIMCE pretende "contribuir al mejoramiento de la calidad y equidad de la educación, obteniendo y comunicando información útil, válida y confiable sobre resultados de aprendizaje del sistema escolar y sus factores asociados. Lo anterior, tanto a nivel nacional, regional y comunal, como de los establecimientos educacionales. El SIMCE realiza sus funciones de acuerdo a lo que establece la normativa legal vigente, y enmarcado en las políticas de educación del MINEDUC y en la ley de presupuesto vigente". 


\subsection{Propósitos del sistema}

El SIMCE no ha experimentado mayores cambios con relación a sus propósitos desde que la Comisión SIMCE entregó sus recomendaciones en 2003; por ello, aún procura:

- monitorear los resultados de aprendizaje del sistema escolar, a nivel nacional e internacional; y orientar decisiones de política educativa, a partir del análisis de factores asociados;

- retroalimentar las prácticas pedagógicas y de gestión de los docentes y directivos, con el fin de mejorar los procesos de enseñanza y aprendizaje;

- $\quad$ promover la rendición de cuentas de los docentes, directivos y sostenedores; y el involucramiento de los padres y apoderados, comunicándoles los resultados de las evaluaciones.

En este sentido, no cabe duda que los resultados recientes de SIMCE y PISA (Mineduc, 2010 y 2011) con relación a los logros alcanzados por los estudiantes de enseñanza básica y media durante la última década (véanse Figuras $\mathrm{N}^{\mathrm{o}} 1,2$ y 3) son un buen ejemplo del impacto

FIGURA N ${ }^{\circ} 1: \quad$ TENDENCIA DE LOS RESULTADOS DE LECTURA EN $4^{\circ}$ BÁSICO

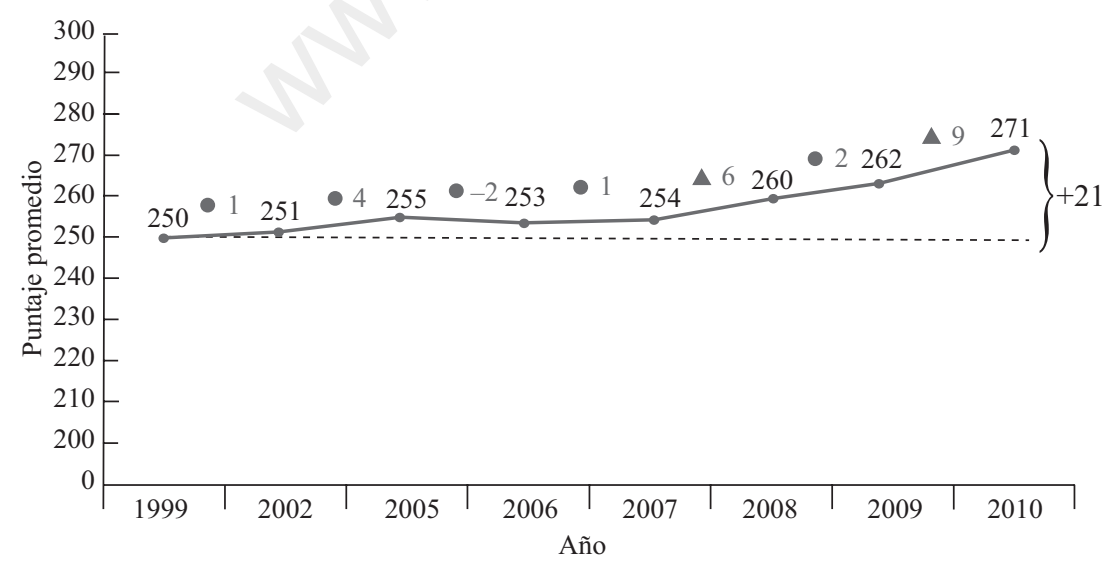

-: Indica que el puntaje promedio es similar al de la evaluación anterior.

$\star$ : Indica que el puntaje promedio es significativamente más alto que el de la evaluación anterior.

+: Indica que la diferencia entre los puntajes del año 1999 y 2010 es estadísticamente significativa. 
FIGURA N ${ }^{\circ}$ 2: $\quad$ TENDENCIA DE LOS RESULTADOS DE EDUCACIÓN MATEMÁTICA EN $4^{\circ}$ BÁSICO.

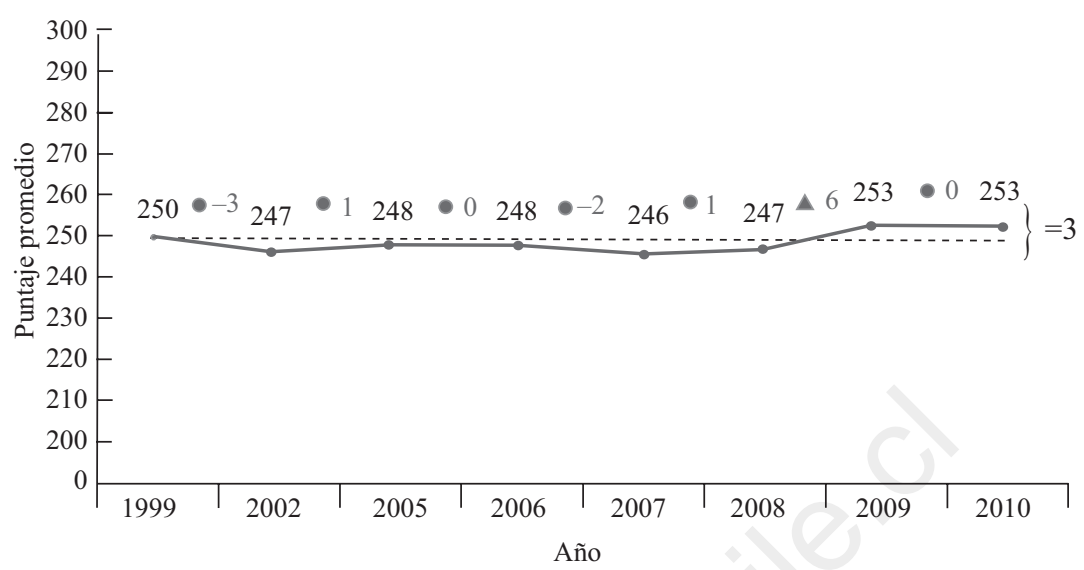

-: Indica que el puntaje promedio es similar al de la evaluación anterior.

^: Indica que el puntaje promedio es significativamente más alto que el de la evaluación anterior.

=: Indica que no hay diferencia significativa entre los puntajes del año 1999 y 2010 .

FIGURA N ${ }^{\circ}$ 3: $\quad$ TENDENCIA DE LOS RESULTADOS DE LECTURA DE LOS ESTUDIANTES DE 15 AÑOS EN PISA.

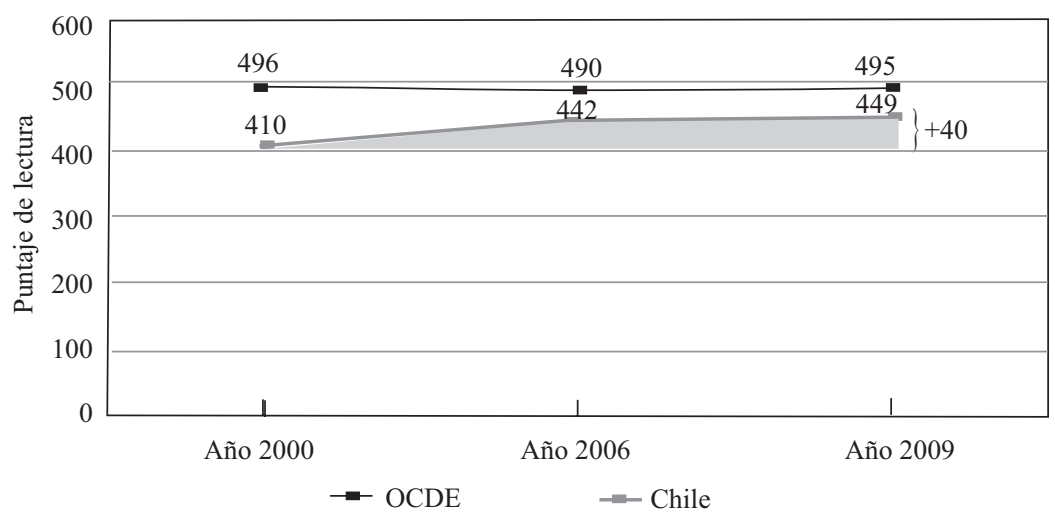


que puede llegar a tener el SIMCE, en la medida que cumple con sus propósitos, dado que aporta evidencia sobre los resultados de las políticas implementadas (campañas, programas educativos, planes de mejoramiento, etc.) y, al mismo tiempo, abre nuevas interrogantes acerca de cómo mejorar los resultados sostenidamente a lo largo del tiempo.

\subsection{Organigrama y funciones}

Actualmente el SIMCE se organiza en dos áreas principales, a saber: el área de pruebas nacionales y el área de estudios internacionales, dependientes de la coordinación nacional y de la coordinación técnica (véase Figura $\mathrm{N}^{\mathrm{o}} 4$ ). El área de pruebas nacionales está conformada por el Equipo de Diseño de Instrumentos, el Equipo de Operaciones de Campo, el Equipo Procesamiento y Análisis de Datos, y el Equipo de Comunicación de Resultados. También forman parte de esta área los proyectos más recientes del SIMCE, a saber: las evaluaciones para estudiantes con necesidades

FIGURA N ${ }^{\circ}$ 4: $\quad$ ORGANIGRAMA DEL SIMCE

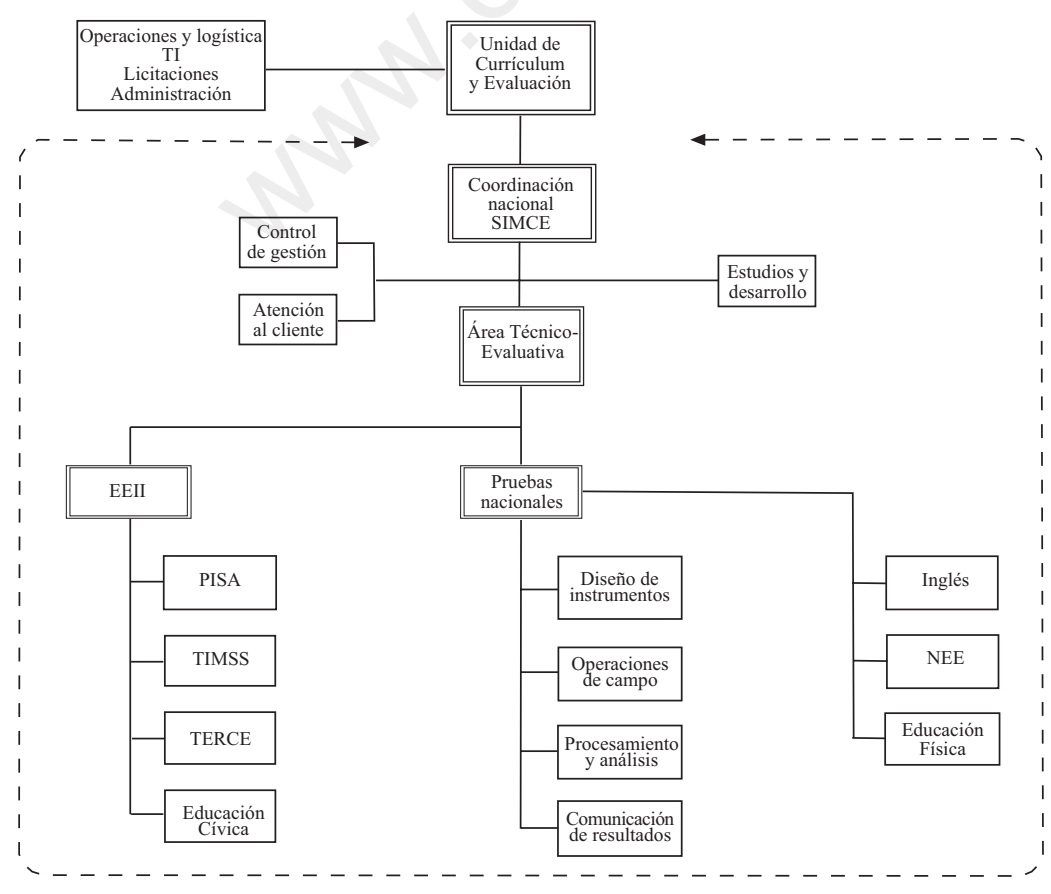


educativas especiales, la evaluación de Educación Física, y la evaluación de Inglés. El área de Estudios Internacionales, por su parte, está conformada por las coordinaciones nacionales de los estudios internacionales en los que participa Chile: PISA, TIMSS, TERCE, y Educación Cívica.

En el área de pruebas nacionales, el Equipo de Diseño de Instrumentos es el encargado de definir los marcos de evaluación y/o especificaciones de las pruebas y cuestionarios; elaborar las preguntas y sus pautas o criterios de corrección. En las evaluaciones internacionales es responsable de la traducción y adaptación de las pruebas. El Equipo de Operaciones de Campo, por su parte, es responsable del diseño operativo de la aplicación nacional y de apoyar la implementación de los operativos nacionales correspondientes a los estudios internacionales. El Equipo Procesamiento y Análisis de Datos se ocupa de realizar los análisis que permiten el cálculo de puntajes de los estudiantes, la determinación del porcentaje de estudiantes correspondiente a cada nivel de logro, y la construcción de los índices estadísticos utilizados para reportar los resultados. El Equipo de Comunicación de Resultados está encargado de que los resultados del SIMCE sean comprendidos y utilizados adecuadamente por los docentes, directivos, padres y apoderados, y sostenedores de los establecimientos. Finalmente, el área de estudios internacionales, realiza la contraparte técnica en la elaboración, aplicación y análisis de las pruebas internacionales en las que participa Chile, a saber, PISA, TIMSS, Educación Cívica y los estudios del Laboratorio Latinoamericano de Evaluación de la Calidad de la Educación (PERCE, SERCE y TERCE).

En cuanto a las áreas de apoyo para el funcionamiento del SIMCE (operaciones y logística, tecnologías de la información, licitaciones y compras, y administración y finanzas) cabe señalar que estas dependen directamente de la Coordinación de la UCE, puesto que también son requeridas por el resto de los componentes de esta (currículum, textos escolares y centros de recursos de aprendizaje).

\section{La transición del SIMCE hacia una nueva institucionalidad}

\subsection{La Ley General de Educación}

La promulgación de la Ley General de Educación (Ley Nº 20.370 de 2009) marcó el comienzo de una serie de cambios a nivel del sistema educativo que modificarán substancialmente el sistema nacional de eva- 
luación. Esta ley establece reformas que afectan de manera directa la institucionalidad y funciones del SIMCE, específicamente en lo que refiere a las evaluaciones de desempeño de los establecimientos educacionales y las mediciones de resultados para determinar el logro de los estándares de aprendizaje, y a la implementación de sistemas de información pública y de rendición de cuentas.

La ley establece la creación de un Sistema Nacional de Aseguramiento de la Calidad de la Educación (véase Figura $\mathrm{N}^{0}$ 5), que deberá encargarse de garantizar el cumplimiento de los estándares de calidad a través de cuatro instituciones. Por una parte, el Ministerio de Educación, organismo que propone las bases curriculares, los programas de estudio y los estándares de calidad, y da apoyo a los establecimientos para su cumplimiento; el Consejo Nacional de Educación, organismo que aprueba las bases, planes y estándares de calidad concebidos por el ministerio. En tercer lugar, la Agencia de Calidad de la Educación, que aparece como la entidad responsable de evaluar e informar sobre la calidad de los establecimientos educacionales, y por último, la Superintendencia de Educación, organismo cuya principal responsabilidad es fiscalizar que los establecimientos cumplan con las normas educacionales y den cuenta pública, cuando sean requeridos.

Según lo señalado en la Ley General de Educación, las principales atribuciones que tendrá la Agencia de Calidad de la Educación para cumplir con sus objetivos serán las siguientes:

- Diseñar e implementar el sistema nacional de evaluación de logros de aprendizaje. Esta medición verificará el grado de cumplimiento de los objetivos generales a través de la medición de estándares de aprendizaje referidos a las bases curriculares nacionales de educación básica y media. La Agencia deberá contar con instrumentos válidos y confiables para dichas evaluaciones, que se apliquen en forma periódica a lo menos en un curso, tanto en el nivel de educación básica como en el de educación media, e informar los resultados obtenidos.

- Coordinar la participación de Chile en mediciones internacionales de aprendizaje de los estudiantes.

- Diseñar e implementar un sistema de evaluación del desempeño de los establecimientos y sostenedores educacionales. Esta evaluación estará basada en estándares indicativos de desempeño de 


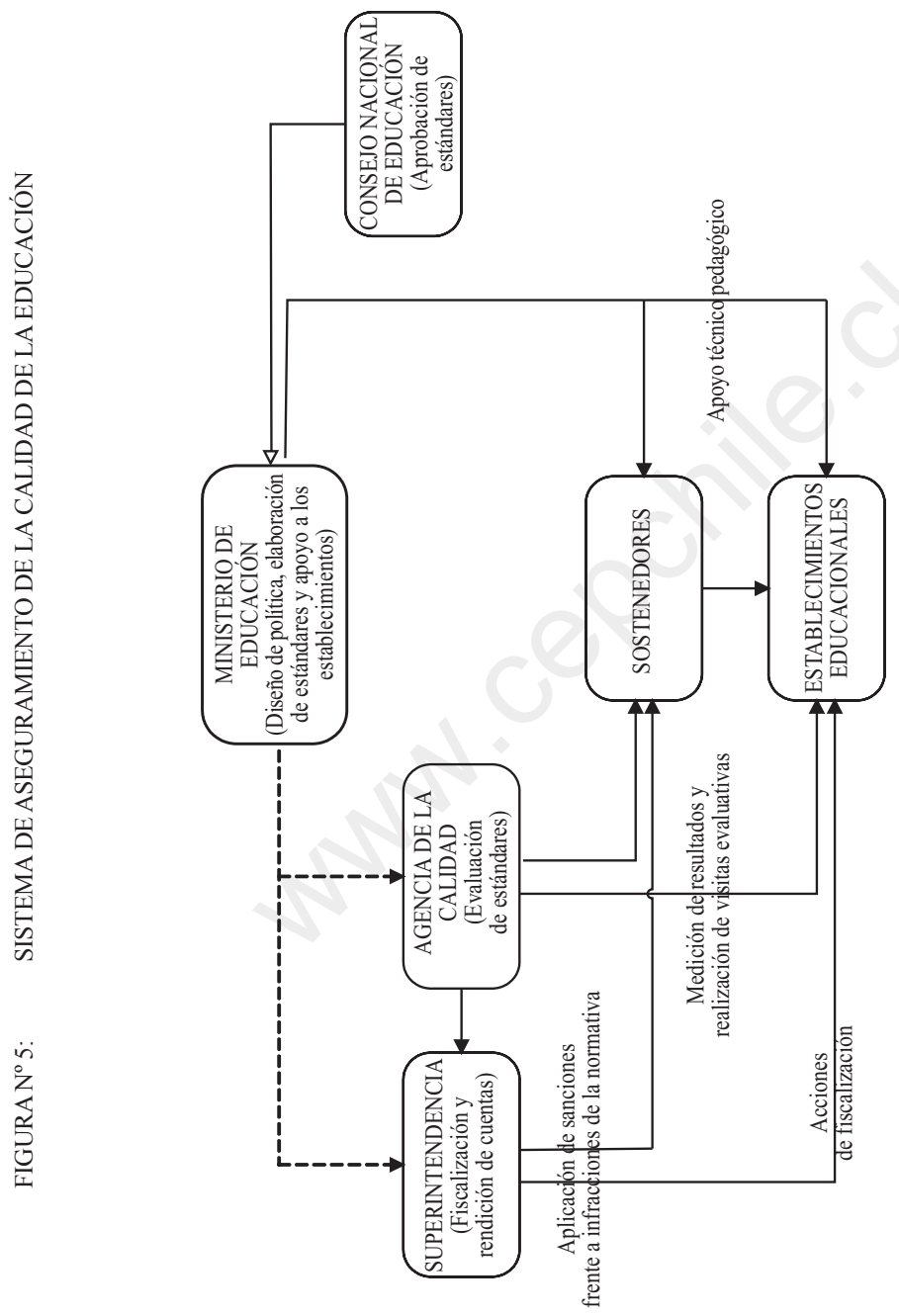


los establecimientos, elaborados por el Ministerio de Educación y aprobados por el Consejo Nacional de Educación.

- Informar públicamente los resultados obtenidos a nivel nacional y por cada establecimiento educacional evaluado.

Sin embargo, la vigencia de la Ley General de Educación depende de la promulgación de otra ley, la Ley de Aseguramiento de la Calidad, actualmente en su último trámite legislativo. En este proyecto de ley se especifican las funciones de la Agencia de la Calidad y de la Superintendencia de Educación, precisándose que la primera será responsable de:

- Llevar a cabo la evaluación de estándares de aprendizaje y otros indicadores de calidad educativa.

- Efectuar la ordenación de establecimientos en función de los resultados de aprendizaje y otros indicadores de calidad educativa.

- Realizar las evaluaciones del desempeño de los establecimientos educacionales, en base a estándares indicativos de desempeño.

- Validar los mecanismos de evaluación de docentes y directivos.

- Informar públicamente los resultados obtenidos a nivel nacional y por cada establecimiento educacional evaluado.

\subsection{Los nuevos desafíos del sistema nacional de evaluación (SIMCE)}

Es evidente, a partir de lo señalado anteriormente que el SIMCE formará parte de la Agencia de la Calidad, por lo que en ese contexto se verá obligado a enfrentar una serie de desafíos para dar respuesta a los requerimientos de la nueva institucionalidad.

En primer lugar será necesario elaborar un nuevo calendario de evaluaciones que contemple la aplicación de pruebas en una mayor cantidad de niveles educacionales y áreas curriculares, así como la recopilación de información relativa a otros indicadores de calidad, para llevar a cabo una ordenación válida y confiable de los establecimientos educacionales (X. Vanni \& J. Bravo, 2010). Este incremento de las evaluaciones adicionalmente permitirá contar con un registro de la trayectoria escolar de cada estudiante, que posibilitaría llevar a cabo mediciones de progreso educativo y/o valor agregado de los establecimientos. En cuanto a los niveles que se deberían incorporar a la evaluación, sería 
recomendable considerar la inclusión de mediciones censales en $2^{\circ} \mathrm{y}$ $6^{\circ}$ año básico, puesto que esto permitiría que todos los establecimientos del país, sin excepción, puedan contar con un diagnóstico de los conocimientos y habilidades de sus estudiantes al comienzo y al final de la enseñanza básica, ya que se debe tener en cuenta que las reformas a aplicarse dentro de los próximos años situarían a $6^{\circ}$ básico como el último nivel de la enseñanza básica. Por otra parte, también será importante enfrentar el desafío de evaluar nuevas áreas de aprendizaje, incorporando áreas que hasta ahora no habían sido abordadas, pero que no por ello son menos importantes. Este solo sería el caso de Escritura y Tecnologías de Información y Comunicación, dado que Educación Física e Inglés fueron incorporadas recientemente a la evaluación.

Otro desafío importante será delinear e implementar acciones orientadas a optimizar la entrega de información a los padres y apoderados, teniendo en consideración sus necesidades concretas de información acerca de la calidad de la educación impartida por los establecimientos educacionales. Para esto será fundamental contar con reportes que integren de manera comprensible y útil toda la información que estará disponible sobre la evaluación de los establecimientos, especialmente su categoría según la ordenación. Además, también será importante planificar cuidadosamente las acciones de difusión de esta información considerando tanto soportes impresos como digitales, de manera de facilitar el acceso de los distintos usuarios.

En el ámbito de la comunicación y difusión, es clave que la entrega de más y mejor información a los padres y apoderados de los estudiantes se complemente con una profundización de la entrega de información a docentes y directivos. Por esto, será fundamental elaborar y comunicar, a la brevedad posible, los estándares de aprendizaje de los estudiantes y los estándares de desempeño de los establecimientos, para que los docentes y directivos cuenten con información pertinente para retroalimentar sus prácticas y puedan dar respuesta a las crecientes demandas externas. Asimismo, sería recomendable adelantar la fecha en que se comunican los resultados de las pruebas nacionales; incorporar indicadores de logro por subáreas o ejes curriculares, en la entrega de resultados; y profundizar los análisis de factores asociados al rendimiento escolar, para que la información entregada a los docentes sea más oportuna y útil. 
Tal como se indicó anteriormente, la ordenación de los establecimientos conlleva varios desafíos técnicos, puesto que se requerirá optimizar las técnicas de recogida de datos de los indicadores de calidad y de nivel socioeconómico, así como desarrollar e implementar una metodología para llevar a cabo una ordenación válida, confiable y útil de los establecimientos educacionales, en base a estándares de aprendizaje y otros indicadores de calidad educativa. En este sentido, será esencial identificar diversos indicadores de calidad educativa y planificar anticipadamente su recopilación, contemplando, entre estos, indicadores de progreso y/o valor agregado en la entrega de resultados; de manera de no continuar posponiendo su utilización para la ordenación de los establecimientos.

Finalmente, es importante tener en consideración que previo a la implementación de la nueva institucionalidad será necesario reevaluar la estructura organizacional del SIMCE, para asegurar que esta pueda dar respuesta eficaz a sus nuevos requerimientos. No obstante, cabe señalar que en este aspecto será fundamental dar énfasis desde un inicio a la captación, formación y retención de recursos humanos competentes y bien calificados, puesto que de esto dependerá en último término la capacidad real del sistema para dar respuesta a las nuevas demandas institucionales (T. Postlethwaite \& T. Kellaghan, 2008). Por otra parte, también será importante diseñar e implementar una estrategia que permita establecer convenios a mediano y largo plazo con los proveedores de productos y servicios más críticos del sistema, vía convenios y/o licitaciones públicas, dado que esto también podría transformarse en una limitante para su crecimiento y expansión.

\section{Comentarios finales}

Los sistemas nacionales de evaluación son un componente característico de los sistemas educativos con alto desempeño (OCDE, 2004b; M. Barber \& M. Mourshed, 2007), por lo que es indudable que la implementación del SIMCE a fines de los 80 constituyó una medida visionaria que anticipó en más de una década políticas educativas que comenzaron a implementarse posteriormente, a nivel internacional. Sin embargo, la evidencia comparada ha demostrado recientemente que el impacto de los sistemas de evaluación puede ser muy limitado si estos no se articulan adecuadamente con otras políticas educacio- 
nales (D. Hopkins, 2007). Por esto, el tránsito del SIMCE a una nueva institucionalidad y su inserción dentro del Sistema de Aseguramiento de la Calidad constituye un gran avance, puesto que lo sitúa como un componente más de un eficaz sistema de rendición de cuentas, y no como la única herramienta disponible para evaluar el desempeño de los estudiantes, los establecimientos, e inclusive de los docentes.

Por último, es importante destacar la capacidad del SIMCE para evolucionar a lo largo del tiempo, adaptándose en más de una oportunidad a nuevas demandas externas. Sin duda, este rasgo ha sido esencial para permitir su permanencia en el tiempo y ha contribuido, en forma determinante, a incrementar su valoración social. No obstante, cabe advertir que esto ha sucedido porque, en general, ha sido posible alcanzar un punto de equilibrio entre elementos de continuidad y cambio, en sus distintas etapas; lo cual debería tenerse muy en cuenta en el tránsito del sistema a una nueva institucionalidad.

\section{REFERENCIAS}

Barber, M. \& M. Mourshed (2007). How the World's Best-Performing School Systems Come Out On Top. Londres, Inglaterra: McKinsey \& Company. Himmel, E. (1997). "Impacto de los Sistemas de Evaluación del Rendimiento Escolar: El Caso de Chile". En B. Álvarez y M. Ruiz-Casares (eds.), Evaluación y Reforma Educativa: Opciones de Politica. PREAL.

Hopkins, D. (2007). Every School a Great School. Open University Press. UK: Berkshire.

Meckes, L. (2003). "SIMCE: Su Desarrollo y sus Desafíos Actuales". Revista Pensamiento Educativo Vol. 33, pp. 160-178, Santiago, Chile.

(2007). "Evaluación y Estándares: Logros y Desafíos para Lograr Impacto en Calidad Educativa". Revista Pensamiento Educativo Vol. 40, pp. 351-371, Santiago, Chile.

Mineduc, Comisión para el Desarrollo y Uso del SIMCE (2003). "Evaluación de Aprendizajes para una Educación de Calidad”. Santiago, Chile.

_ Unidad de Currículum y Evaluación (2010). "Informe Nacional de Resultados de PISA 2009". Santiago, Chile.

__ Unidad de Currículum y Evaluación (2011). "Resultados Nacionales SIMCE 2010". Santiago, Chile.

Montt, P. et al. (2010). "Memoria de la Unidad de Currículum y Evaluación 2006-2010: Funciones, Organización y Agenda". Documento Interno Santiago, Chile: Ministerio de Educación.

OCDE (2004a). "What Makes Schools System Perform? Seeing School System Trough the Prism of PISA". París: OCDE. (2004b). "Revisión de Políticas Nacionales de Educación. Organización para la Cooperación y el Desarrollo Económico". Chile, Francia: OCDE. 
Postlethwaite, T., \& T. Kellaghan (2008). "National Assessment of Educational Achievement". Educational Policy Series. París: International Academy of Education and the International Institute for Educational Planning.

Román, M. (1999). "Usos Alternativos del SIMCE: Padres, Directores y Docentes". Documentos N ${ }^{\circ}$ 5, CIDE, Santiago, Chile.

Vanni, X. \& J. Bravo (2010). "En Búsqueda de una Educación de Calidad para Todos: El Sistema Nacional de Aseguramiento de la Calidad". En S. Martinic y G. Elacqua (eds.), ¿Fin de Ciclo? Cambios en la Gobernanza del Sistema Educativo. Santiago, Chile: UNESCO/PUC. 